\title{
Treating liver cancer with antibiotics?
}

\author{
Hui MAO, Xue-zhu FENG, Shou-hong GUANG*
}

Acta Pharmacologica Sinica (2013) 34: 989-990; doi: 10.1038/aps.2013.102

$\mathrm{O}$ besity is one of the most prevalent health concerns over the past few decades. Besides diabetes and cardiovascular disease, obesity is also connected to multiple types of cancers ${ }^{[1,2]}$. However, the mechanism underlying obesity-related cancers is unclear. Previous research found that obesity alters the microbiota grown in the gastrointestinal tract ${ }^{[3]}$, where the microbes produce inflammatory metabolites ${ }^{[4]}$. And obesity-induced inflammation is involved in liver cancer ${ }^{[5]}$. Recently, Yoshimoto et al uncovered the missing link, by which obesity promotes the production of proinflammatory and carcinogenic bile acids via shaping gut microbiota, and leads to hepatic inflammation and hepatocellular carcinoma (HCC) ${ }^{[6]}$ (Figure 1).

In mammals, bile acids are secreted into the gut lumen in high millimolar concentrations. They are converted to various secondary bile acids by microbes that are most densely colonized in the distal gut ${ }^{[7]}$. Secondary bile acids, including deoxycholic acid (DCA), are uptake by enterohepatic circulation to the liver. Feeding mice with high-fat diet (HFD) raised serum levels of DCA, whereas antibiotic treatment reduced the DCA levels ${ }^{[6]}$. Yoshimoto and colleagues found that feeding mice with HFD dramatically increased the percentage of Gram-positive bacteria in the intestinal tract. In particular, strains belonging to

School of Life Sciences, University of Science and Technology of China, Hefei 230027, China Correspondence to: Prof Shou-hong GUANG (sguang@ustc.edu.cn)
Clostridium cluster XI were substantially increased by HFD. These strains are essential to perform 7a-dehydroxylation that converts primary bile acids to DCA.

DCA causes DNA damage and is involved in the progression of colorectal cancers. In response to DNA damage, cells either age by senescence or selfdestruct via apoptosis if the damage cannot be easily repaired. Although senescent cells can launch the cell-cycle arrest and halt cell replication, they remain metabolically active, and change gene expression, produce pro-inflammatory signaling proteins. In hepatic stellar cells (HSCs), DCA provokes the senescence-associated secretory phenotype (SASP), by which cells release several pro-inflammatory cytokines, chemokines, tissue-remodeling enzymes, and

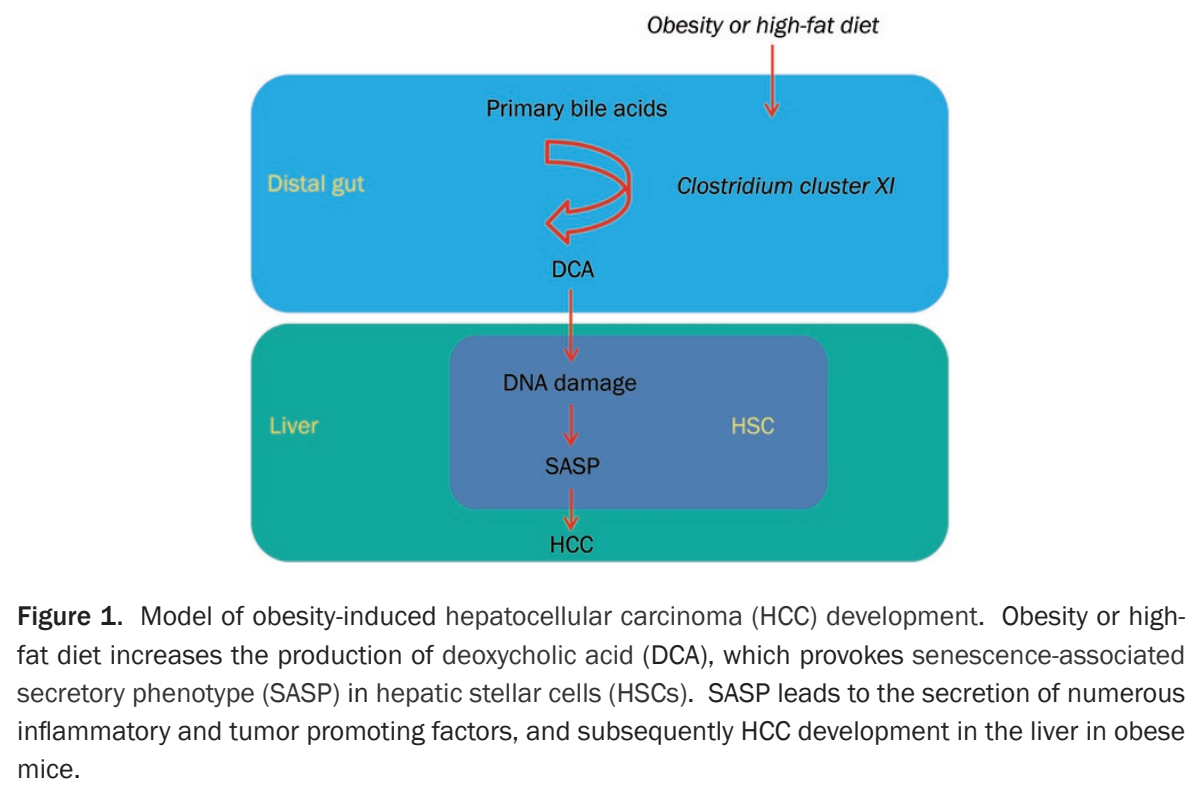

Figure 1. Model of obesity-induced hepatocellular carcinoma (HCC) development. Obesity or highfat diet increases the production of deoxycholic acid (DCA), which provokes senescence-associated secretory phenotype (SASP) in hepatic stellar cells (HSCs). SASP leads to the secretion of numerous inflammatory and tumor promoting factors, and subsequently HCC development in the liver in obese mice.

tumor-promoting factors in the liver ${ }^{[8,9]}$. SASP plays essential roles in promoting the obesity-associated hepatocellular carcinoma (HCC) in mice after exposure to chemical carcinogens that cause DNA damage ${ }^{[8,10]}$. On the other hand, a lack of SASP inducer or depletion of senescent HSCs can efficiently prevent the development of hepatocellular carcinoma in obese mice ${ }^{[6]}$. IL-1 $\beta$ is an upstream regulator of SASP, and is induced in senescent HSCs by HFD. Mice lacking the $I L-1 \beta$ gene reduced the expression of SASP factors in activated HSCs. Meanwhile, the number and size of liver tumors are significantly decreased in $I L-1 \beta^{-/-}$mice. These findings in mice can be extended to human beings. Adding recombinant IL-1 $\beta$ to cultured primary human HSCs induced 
the expression of $I L-6$ and $I L-8$. The signs of cellular senescence and SASP were also observed in HSCs in HCC in nonalcoholic steatohepatitis patients.

Notably, Yoshimoto and colleagues revealed several lines of evidence that blocking DCA production efficiently prevents the progression of hepatocellular carcinoma in obese mice: (1) treating mice with the oral antibiotic cocktail or vancomycin to kill Gram-positive bacteria led to a pronounced reduction of liver cancer; (2) supplementing antibiotics-treated animals with DCA promoted carcinogenesis; (3) the expression of baij gene, which is involved in 7a-dehydroxylation of bile acids, was drastically increased in mice fed HFD, albeit it was reduced by vancomycin. These findings provide valuable insight into the mechanism of obesity-related liver cancer and open up new possibilities for control of HCC.

It is worth noticing that residual hepatocellular carcinoma still exists after the ablation of HSCs by antibiotic treatment. Moreover, in contrast to Yoshimoto's work, intestinal Gram-negative, but not Gram-positive, bacteria have been reported to promote HCC development when induced by a different carcinogen in obese mice. Senescence induction has also been shown to inhibit the growth of several carcinoma cell lines. Therefore, further study of the molecular mechanisms linking gut microbial metabolites to SASP is required for the battle against obesity-associated HCC.

Bile acids have been implicated in a variety of human diseases, but how microbe influences bile acids metabolism is not clarified. Moreover, the byproducts are not only passive carcinogens, but also active signaling molecules, which regulate host lipid and glucose homeostasis, as well as the synthesis of the bile acids themselves. Therefore, it is intriguing to elucidate the metabolism, functions, and regulations of bile acids. Gut microbiota plays important roles in many essential processes, including vitamin and amino acid biosynthesis, dietary energy harvest, and immune development. Numerous factors are able to change the gut microbial community, including diet, disease, and antibiotics, yet the health implications in human are unclear. Developing innovative technologies to systematically characterize the composition of gut bile acids could enable us to better understand the host-microbe interactions and the association with human diseases, for example, obesity and cancer ${ }^{[7]}$. Yoshimoto and colleagues' work paved a new way to study microbial metabolism and tumor microenvironment, as well as risk assessment, diagnostics and therapeutic interventions.

1 Calle EE, Kaaks R. Overweight, obesity and cancer: epidemiological evidence and proposed mechanisms. Nat Rev Cancer 2004; 4: 579-91.

2 Khandekar MJ, Cohen P, Spiegelman BM. Molecular mechanisms of cancer development in obesity. Nat Rev Cancer 2011; 11: 886-95.

3 Greenblum S, Turnbaugh PJ, Borenstein E. Metagenomic systems biology of the human gut microbiome reveals topological shifts associated with obesity and inflammatory bowel disease. Proc Natl Acad Sci U S A 2012; 109: 594-9.

4 Fei N, Zhao L. An opportunistic pathogen isolated from the gut of an obese human causes obesity in germfree mice. ISME J 2013; 7: 880-4.

5 Sun B, Karin M. Obesity, inflammation, and liver cancer. J Hepatol 2012; 56: 704-13.

6 Yoshimoto S, Loo TM, Atarashi K, Kanda H, Sato $\mathrm{S}$, Oyadomari S, et al. Obesity-induced gut microbial metabolite promotes liver cancer through senescence secretome. Nature 2013; 499: 97 101.

7 Ridlon JM, Kang DJ, Hylemon PB. Bile salt biotransformations by human intestinal bacteria. J Lipid Res 2006; 47: 241-59.

8 Kuilman T, Peeper DS. Senescence-messaging secretome: SMS-ing cellular stress. Nat Rev Cancer 2009; 9: 81-94.

9 Friedman SL. Hepatic stellate cells: protean, multifunctional, and enigmatic cells of the liver. Physiol Rev 2008; 88: 125-72.

10 Collado M, Serrano M. Senescence in tumours: evidence from mice and humans. Nat Rev Cancer 2010; 10: 51-7. 